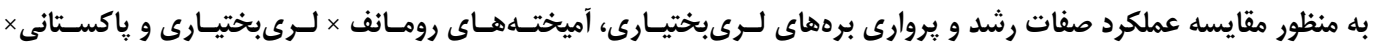

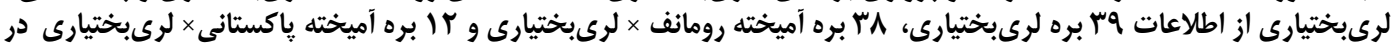

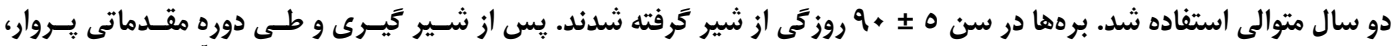

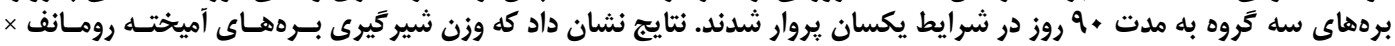

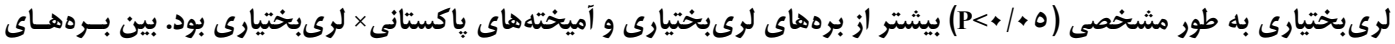

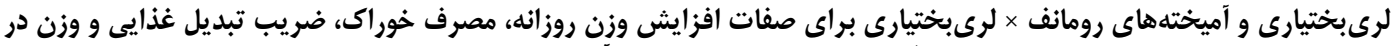

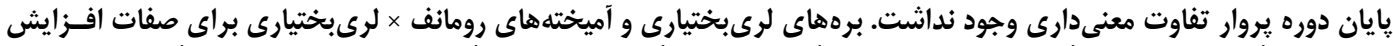

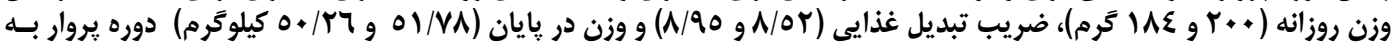

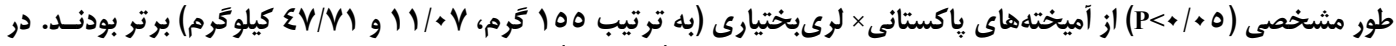

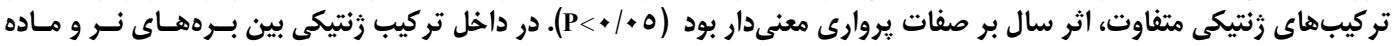

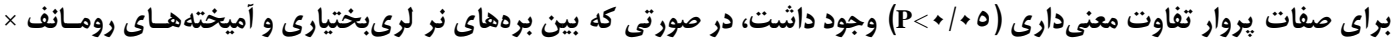

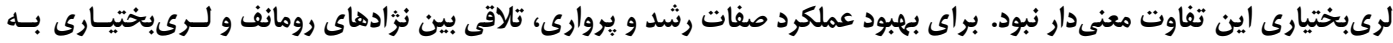

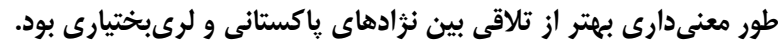

وازههاى كليدى: رشد، يروار، آميختهَّىى، رومانف، پاكستانى، لرىبختيارى

حاصل از تلاقى شال× زل زل از نزاد خالص به طور معنى دادارى

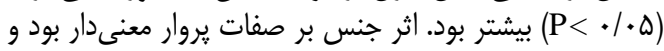

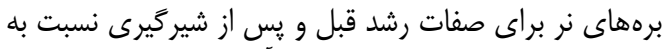

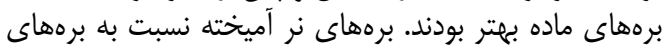

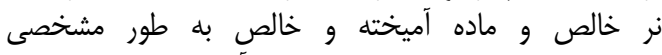

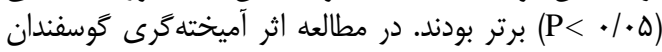

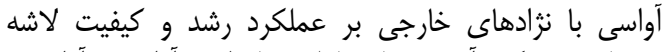

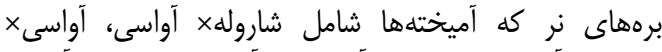

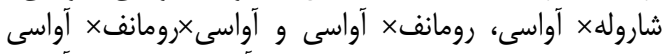

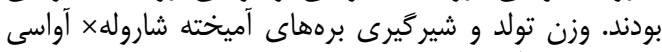

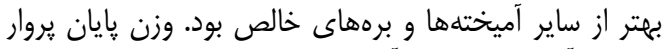

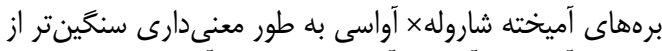

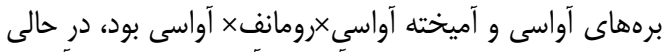

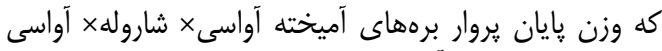

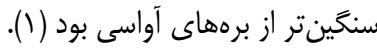

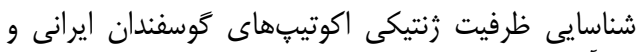

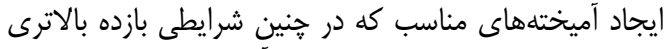

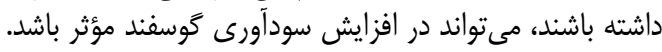

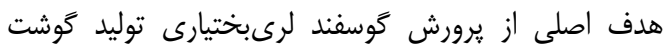

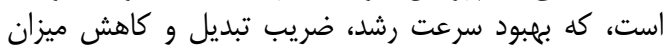

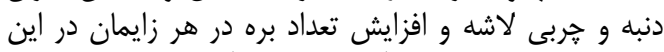

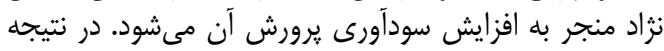

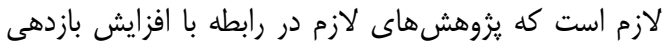

مقدمه

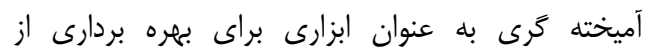

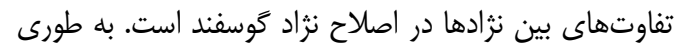

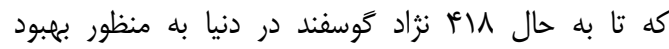

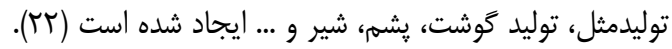

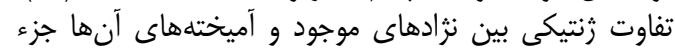

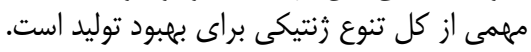

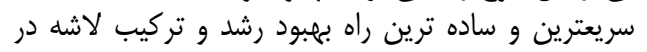

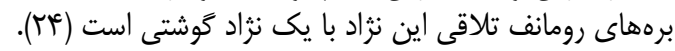

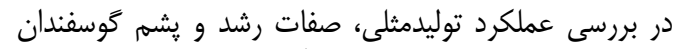

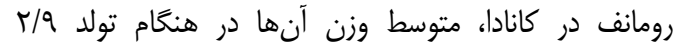

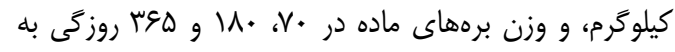

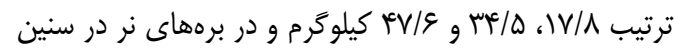

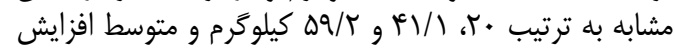

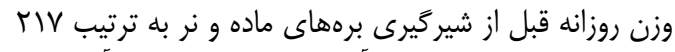

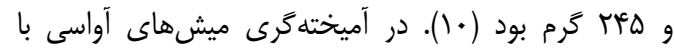

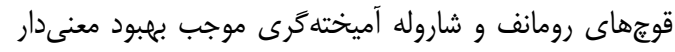

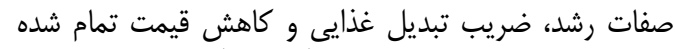

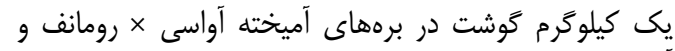
آواسى × شاروله شده است (19).

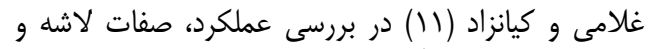

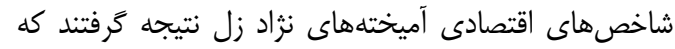

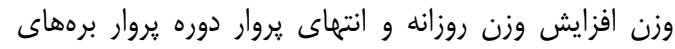


ماده خشك بود. ميزان غذاى مصرفى هر بره، از كسر درائ

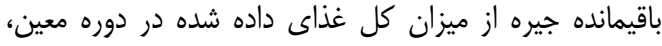

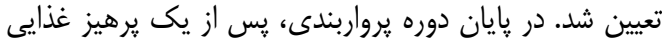

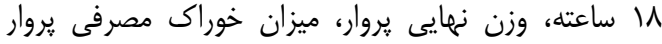

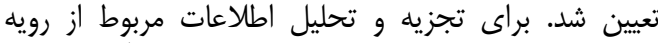

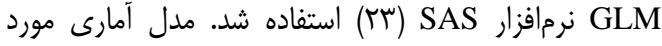

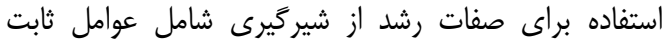

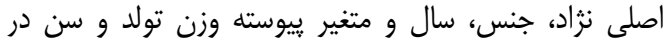

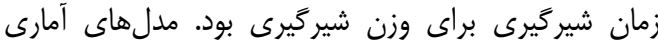
براى صفات بروارى بشرح ذيل بودي

$y_{i j k l}=\mu+A_{i}+B_{j}+C_{k}+b\left(X_{i j k l}-X_{\circ 000}\right)+e_{i j k l}$

$y_{i j k l}=\mu+A B C_{i j k}+b\left(X_{i j k l}-X_{0000}\right)+e_{i j k l}$

در اين مدلها:

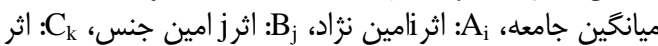

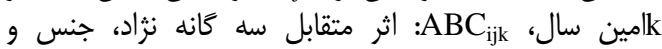

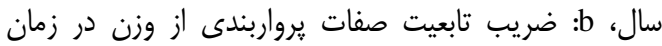

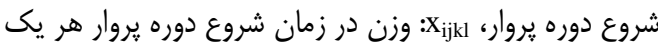

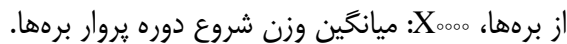

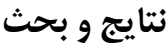

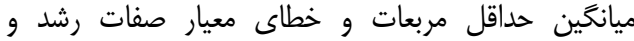

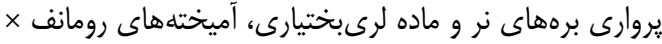

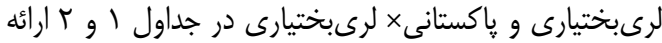

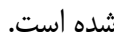

اقتصادى از طريق افزايش تعداد بره و بهبود سرعت رشد،

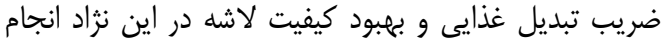

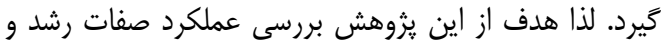

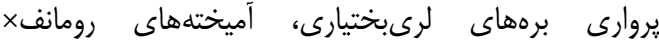
لرىبختيارى و پاكستانى × لرى بختيارى بود.

مواد و روشها

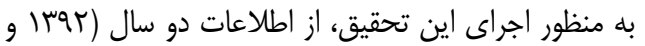

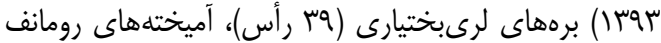

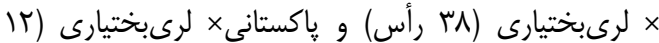

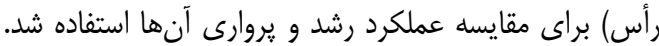

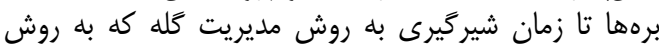

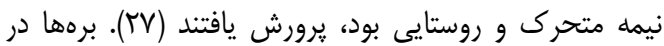

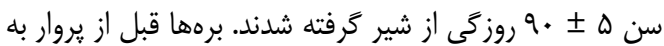

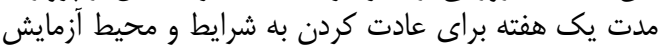

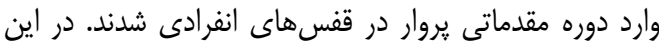

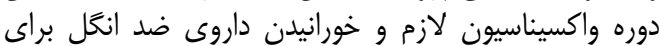

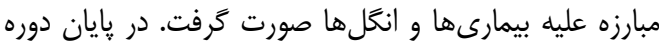

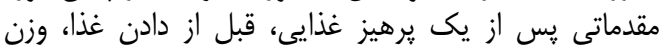

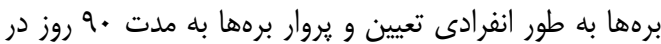

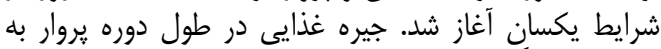

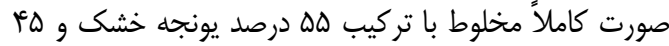

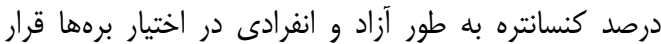
كرفت. ميزان ماده خشك جيره

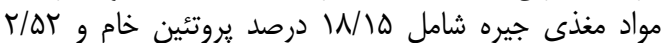

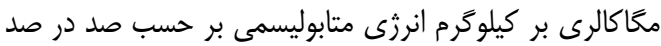

جدول 1- ميانگين حداقل مربعات و خطاى معيار صفات رشد و يروارى برههاى لرى لريختيارى، آميختهاى رومانف × لرى بختيارى و ياكستانى ×

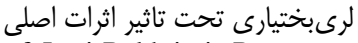

Table 1. Least square means and standard error of growth and feedlot traits of Lori-Bakhtiari, Romanov $\times$ LoriBakhtiari and Pakestani $\times$ Lori-Bakhtiari crossbred lambs influenced by main effects

\begin{tabular}{|c|c|c|c|c|c|c|c|}
\hline وزن درو يايان دوره (كيلوگرم) & وزي يروار (كيلوكرم) & افزايش وزن روزانه & وزن شيرگيرى & (كيلوَّم) & تعداد & & اثر \\
\hline$\Delta \backslash / V \wedge \pm \cdot / \Delta \Delta^{\mathrm{a}}$ & $r T / V V \pm \cdot / q \varphi^{a}$ & $r \Delta \& \pm \lambda^{a}$ & $T V / \& \Delta \pm \cdot / \not V^{a}$ & 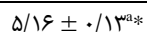 & rq & لرىبختيارى & \multirow{3}{*}{ نزراد } \\
\hline$\Delta \cdot / R \Phi \pm \cdot / \Delta \gamma^{\mathrm{a}}$ & 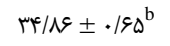 & $r \cdot \mu \pm \lambda^{b}$ & $M / \Delta \cdot \pm \cdot / M^{b}$ & $f / 9 \Delta \pm \cdot / 1 \mu^{\mathrm{a}}$ & 山 & رومانف ×لرىبختيارى & \\
\hline $\mid r / V) \pm \cdot / 9 r^{b}$ & $r / \Delta \Delta \pm 1 / 1 \cdot{ }^{a}$ & $r q \cdot \pm 1 f^{\mathrm{a}}$ & $r V / q r \pm 1 / r r^{a}$ & $\Delta / \wedge q \pm \cdot / \pi^{b}$ & ir & پاكستانى ×لرى بختيارى & \\
\hline$\leftarrow \varepsilon / F r \pm \cdot / \Delta r^{\mathrm{a}}$ & $r r / F V \pm \cdot / \mathscr{G} F^{\mathrm{a}}$ & $r a r \pm \Lambda^{\mathrm{a}}$ & $r \cdot / 9 q \pm \cdot / v \cdot^{a}$ & $\omega / \cdot \vee \pm \cdot / / r^{\mathrm{a}}$ & f. & اول & \multirow{2}{*}{ سال } \\
\hline$\leftarrow q / \sim q \pm \pm \cdot / \Delta \Delta^{b}$ & $\Gamma \Psi / \& \Delta \pm \cdot / 9 \varphi^{\mathrm{a}}$ & $r \Delta T \pm \Lambda^{b}$ & $r V / \widetilde{q} \pm \cdot / V \varphi^{b}$ & $\Delta / q \cdot \pm \cdot / / r^{b}$ & pq & دوم & \\
\hline$\Delta r / \Delta V \pm \cdot / \Delta \cdot^{a}$ & 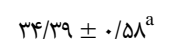 & $r \Lambda I \pm v^{a}$ & $r N / r T \pm \cdot / N{ }^{a}$ & $\Delta / r \Lambda \pm \cdot / / r^{a}$ & W & نر & \multirow[b]{2}{*}{ جنس } \\
\hline 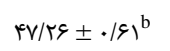 & $r / / r \pm \pm \cdot / 9 \Lambda^{b}$ & $r 9 \Delta \pm \lambda^{\mathrm{a}}$ & $r q / v^{u} \pm \cdot / q r^{\mathrm{a}}$ & $\Delta / \tau \Lambda \pm \cdot / / f^{\mathrm{a}}$ & عq & ماده & \\
\hline
\end{tabular}


جدول r- ميانكين حداقل مربعات و خطاى معيار صفات يروارى برههاى لرىبختيارى، آميختههاى رومانف × لرىبختيارى و پِاكستانى ×

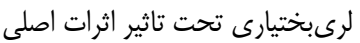

Table 2. Least square means and standard error of feedlot traits of Lori-Bakhtiari, Romanov $\times$ Lori-Bakhtiari and Pakistani $\times$ Lori-Bakhtiari crossbred lambs influenced by main effects

\begin{tabular}{|c|c|c|c|c|c|c|c|}
\hline بازده غذايى دوره & ضريب تبديل دوره & مصرف خوراك دوره & افزايش وزن روزانه & يروار (كيلوَرم) & تعداد & & اثر \\
\hline$|r / r F \pm \cdot / F|^{a}$ & $N / Q T \pm \cdot / r^{a}$ & $1 / 94 \pm . / \cdot 1^{\mathrm{a}}$ & $r \cdot . \pm q^{\mathrm{a}}$ & $I V / a \vee \pm \cdot / \Delta \Delta^{\mathrm{a}}$ & rq & لرىبختيارى & \multirow{3}{*}{ 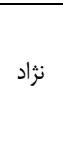 } \\
\hline $11 / 9 \pm \cdot / 4 r^{a b}$ & $N / 9 \Delta \pm \cdot / \kappa^{\cdot a}$ & $1 / \Delta \Lambda \pm \cdot / \cdot 1^{c}$ & $\operatorname{Mf} \pm q^{\mathrm{a}}$ & $\mid \varepsilon / f \Delta \pm \cdot / \Delta \gamma^{\mathrm{a}}$ & r & رومانف×لرى بختيارى & \\
\hline $1 \cdot / F^{A} \wedge \pm \cdot /\left.V\right|^{\mathrm{b}}$ & $11 / \cdot v \pm \cdot / 8 \Lambda^{\mathrm{b}}$ & $1 / \& \Delta \pm \cdot / \cdot r^{b}$ & $10 \Delta \pm 11^{b}$ & $1 w / a \cdot \pm \cdot / 9 r^{b}$ & ir & ياكستانى لالرىبختيارى & \\
\hline$|r /| V \pm \cdot / \epsilon^{a}$ & $q / r \varepsilon \pm \cdot / \mu^{\mathrm{a}}$ & $1 / 1 \varepsilon \pm . / \cdot 1^{\mathrm{a}}$ & 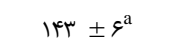 & $|r / 9| \pm \cdot / \Delta r^{a}$ & f. & 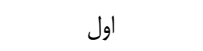 & \multirow{2}{*}{ سال س } \\
\hline $1 . / 9 V \pm . / F r^{b}$ & $q / V V \pm \cdot / r q^{a}$ & $1 / 9 \Delta \pm \cdot / \cdot 1^{b}$ & $r M \pm \xi^{b}$ & $19 / 9 \cdot \pm \cdot / \Delta \Delta^{b}$ & pq & دوم & \\
\hline $\mid r / F V \pm \cdot / r v^{a}$ & $N \cdot r \pm \cdot / r \Delta^{a}$ & $1 / \Delta \mathrm{V} \pm \cdot / \cdot \mathrm{v}^{\mathrm{a}}$ & $r \cdot 9 \pm q^{\mathrm{a}}$ & $\mid N / V G \pm \cdot / \omega \cdot^{a}$ & $\Delta r$ & 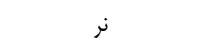 & \multirow[b]{2}{*}{ 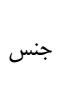 } \\
\hline$q / q \vee \pm \cdot / 4 q^{b}$ & $I N / r \pm \cdot / F^{b}$ & $1 / \Delta f \pm \cdot / \cdot r^{\mathrm{a}}$ & $1 a \cdot \pm v^{b}$ & $\left.|r / F \Delta \pm \cdot| \xi\right|^{b}$ & ع & ماده & \\
\hline
\end{tabular}

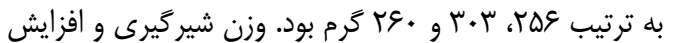

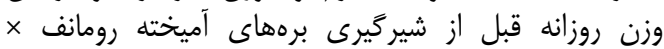

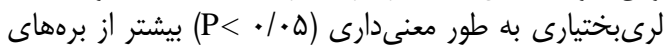

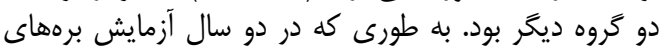

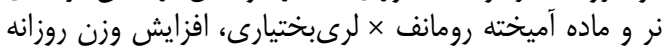

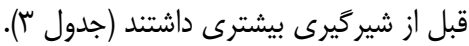

اثر زنوتيي بره بر صفات وزن تولد، وزن شيرگيرى و

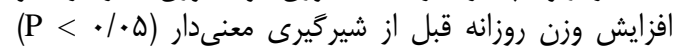

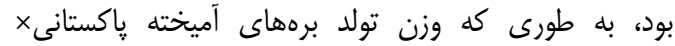

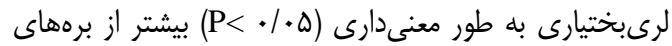

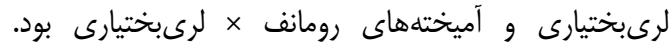

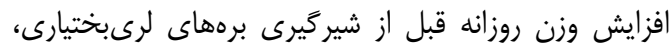

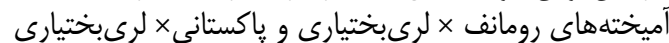

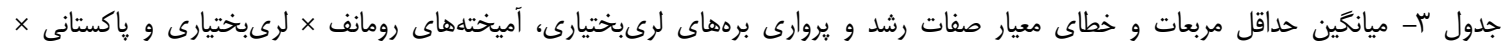
لرىبختيارى تحت تاثير اثرات متقابل مرات

Table 3. Least square means and standard error of growth and feedlot traits of Lori-Bakhtiari, Romanov $\times$ Lori-Bakhtiari and Pakistani $\times$ Lori-Bakhtiari crossbred lambs influenced by interaction effects

\begin{tabular}{|c|c|c|c|c|c|c|c|c|}
\hline 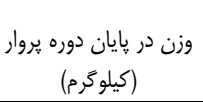 & دوره يروار (كيلوگرم) & افنا شير كيرى (كَرم) & وزن شيرَيرى & (كيلوَرم) & تعداد & سال س & جنس ا & 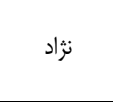 \\
\hline$F_{\Delta} \Delta / \mathrm{FV}^{\mathrm{q}} \pm 1 / \cdot 0^{\mathrm{e}}$ & $r \cdot / q \mid \pm 1 / / \Lambda^{c}$ & $r \& T \pm 10^{b c d}$ & $\Gamma V / \wedge \Delta \pm I / r^{b b c}$ & $r / \wedge q \pm \cdot / r \Delta^{b c d}$ & 1. & اول & \multirow{2}{*}{ ماده } & \multirow{4}{*}{ رى بختيارى } \\
\hline$\Delta F / a q \pm 1 / V \cdot{ }^{a b}$ & $r q / q r \pm 1 / q \omega^{b c}$ & $r r \cdot \pm r r^{d}$ & $r q / q q \pm r / \notin q q c$ & $\Delta / \cdots \pm \cdot / q^{a b c d}$ & f & دوم & & \\
\hline$\Delta \cdot / q \Gamma \pm 1 / . .{ }^{c d}$ & $r T / V g \pm 1 / \backslash \Lambda^{b c}$ & $r q v \pm 10^{a b}$ & $r v / \cdot 9 \pm 1 / r \cdot{ }^{a}$ & $r / q \cdot \pm \cdot / r b^{b c d}$ & 1. & اول ال & \multirow{2}{*}{ 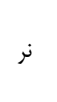 } & \\
\hline$\Delta V / \Lambda r \pm \cdot / \Lambda r^{a}$ & $r \Delta / \wedge \Delta \pm \cdot / \Omega \Lambda^{\mathrm{a}}$ & 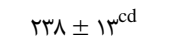 & $r g / 1 V \pm 1 / \cdot \wedge^{c}$ & $\Delta / \Delta V \pm \cdot / r^{\mathrm{a}}$ & 10 & دوم & & \\
\hline$f \cdot / q \& \pm 1 /\left.\psi^{f}\right|^{\mathrm{f}}$ & $r T / . f \pm 1 / 9 \Lambda^{b c}$ & $r \Lambda \Delta \pm \pi r^{\mathrm{abcd}}$ & $r q / v \Delta \pm 1 / M^{b c}$ & 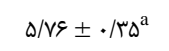 & $\Delta$ & اول اول & ماده & \multirow{3}{*}{ 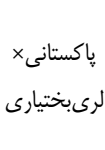 } \\
\hline 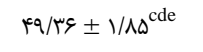 & $r N / q \Lambda \pm r / T F^{c}$ & $r \Delta S \pm r \Lambda^{b c d}$ & $r V / r^{q} \pm r / \kappa^{b c}$ & $s / 1 \cdot \pm \cdot / F \Delta^{\mathrm{a}}$ & r & اول & & \\
\hline$\Delta r / Q \Lambda \pm 1 / \Delta \Lambda^{b c}$ & $r \Psi / \cdot \Delta \pm 1 / \wedge \gamma^{a b c}$ & $r \Delta \Delta \pm r \epsilon^{b c d}$ & $r V / V \cdot \pm r / \cdot^{b c}$ & $\Delta / q \Delta \pm \cdot / / q^{a b}$ & f & 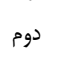 & نر & \\
\hline$F \Delta / V^{e} \pm 1 / r^{e}$ & $r T / r \cdot \pm 1 / \Delta r^{a b c}$ & $r \cdot c \pm r \cdot{ }^{a b}$ & $\Gamma / \& \backslash \pm 1 / v \Delta^{a b}$ & $r / q \cdot \pm \cdot / r^{c d}$ & 9 & اول اول & \multirow{2}{*}{ ماده } & \multirow{4}{*}{ رومبختيارى } \\
\hline $0 \cdot 1 \cdot 9 \pm \cdot / 9 \Delta^{\mathrm{cd}}$ & $r r / q 1 \pm 1 / / r^{\mathrm{ab}}$ & $r r^{\prime} \pm 1 f^{\mathrm{bd}}$ & $r q / q^{2} \pm 1 / \pi r^{b}$ & $\Delta / 19 \pm \cdot / \pi^{\mathrm{abc}}$ & 11 & دوم & & \\
\hline$\kappa N / \cdot V \pm 1 / \Gamma^{2} \gamma^{\mathrm{de}}$ & $r q / \wedge \notin \pm V / \Delta f^{a}$ & $r F I \pm r \cdot{ }^{a}$ & $r F / \wedge q \pm 1 / / c^{a}$ & $r / \Psi \Lambda \pm \cdot / r^{d}$ & 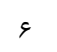 & اول & \multirow[b]{2}{*}{ نر } & \\
\hline$\Delta \varepsilon / \mathrm{W} \pm \cdot / \mathcal{N} \mathcal{F}^{\mathrm{a}}$ & $r g / 4 q \pm . / 9 \gamma^{a}$ & $r \wedge q \pm \| r^{b}$ & $r \cdot / r a \pm 1 / q^{b}$ & $\Delta / r^{a} \pm \cdot / r \cdot{ }^{a b c}$ & 10 & دوم & & \\
\hline
\end{tabular}




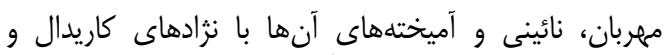

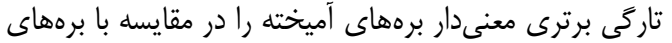

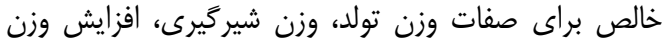

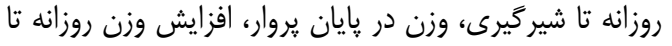

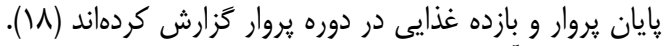

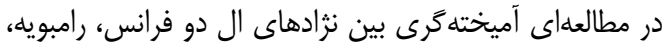

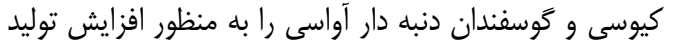

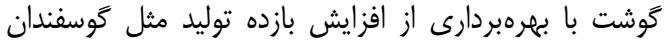

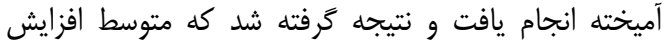

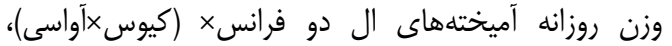

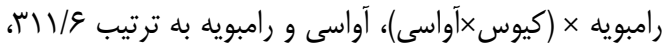
r/T/T

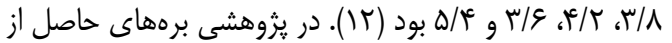

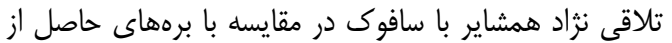

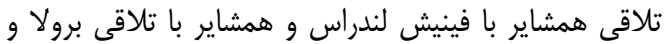

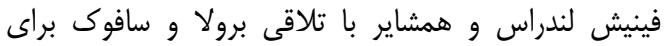

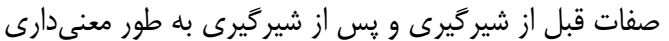

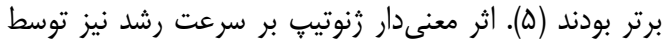

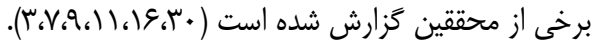

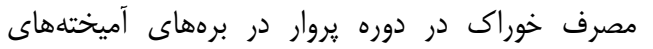

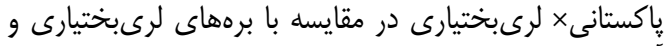

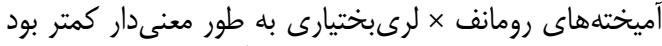

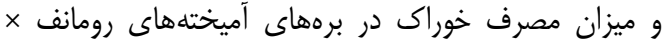

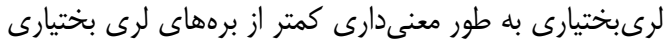

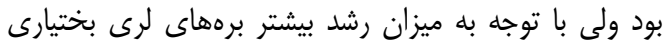

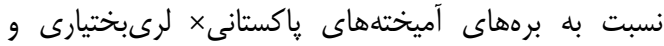

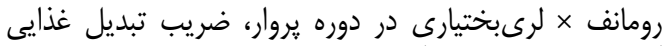

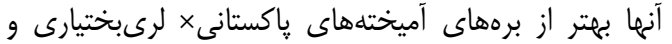

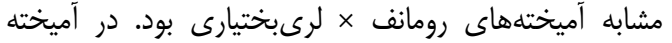

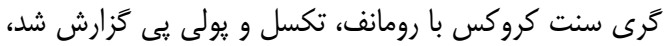

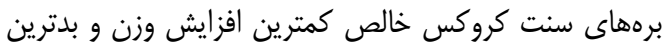

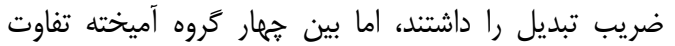

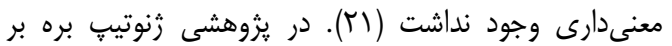

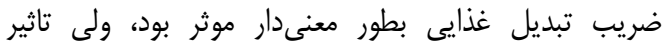

معنى دارى بر مصرف خوراك نداشت (سار)

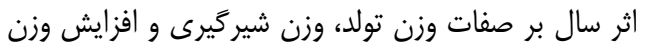

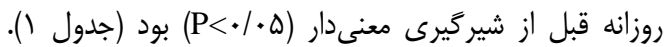

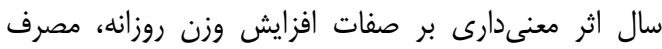

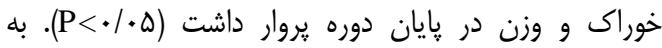

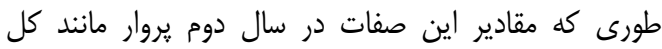

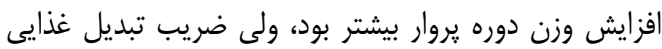

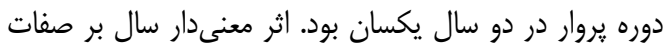

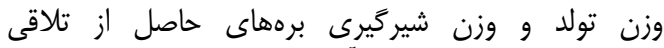

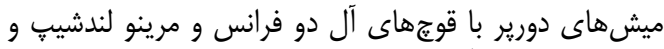

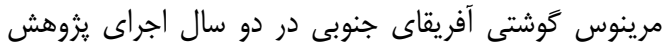

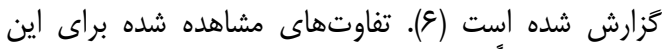

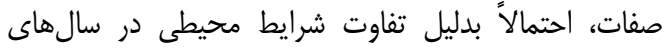

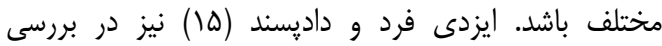
عملكرد بروار برههاى قزل و مهربان و آميختههاى آنها دادئ
وزن بدن نزادهاى مختلف كَوسفندان تحت تأثير قالب

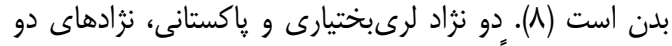

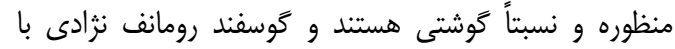

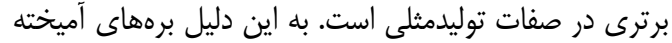

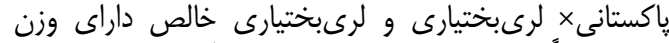

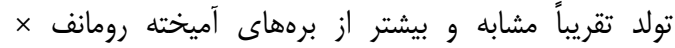

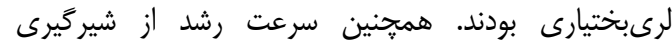

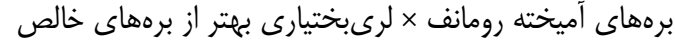

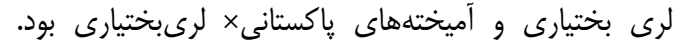

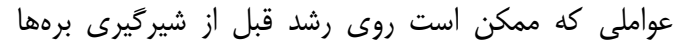

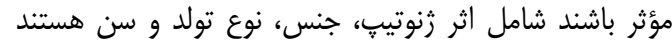

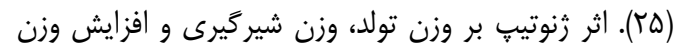

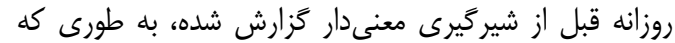

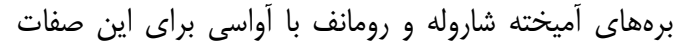

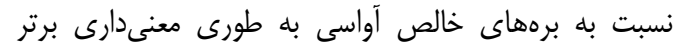

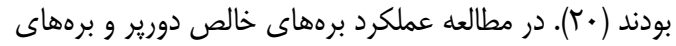

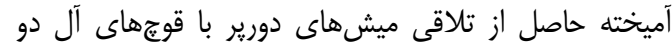

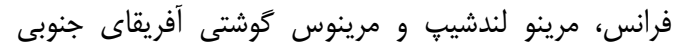

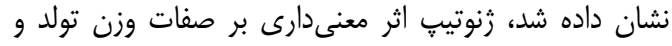

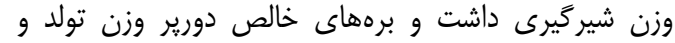

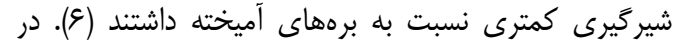

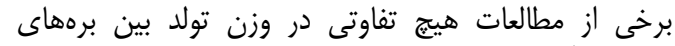

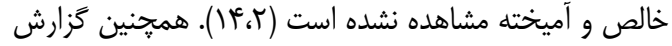

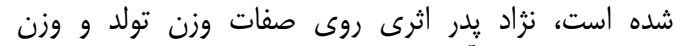

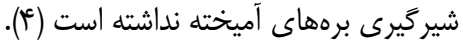

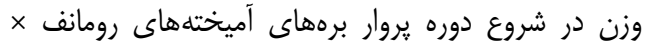

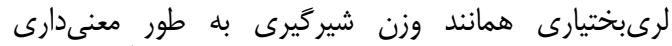
(P</.0)

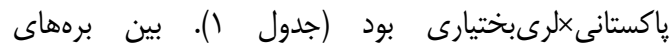

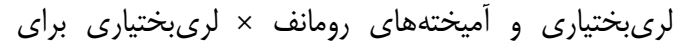

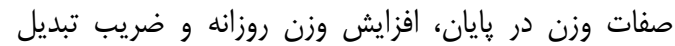

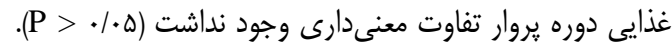

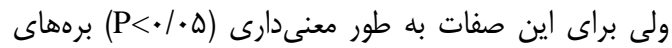

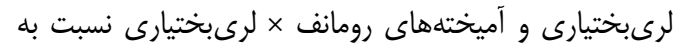

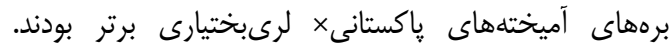

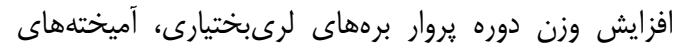

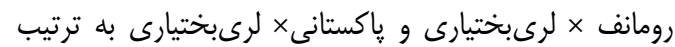

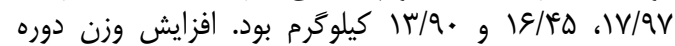

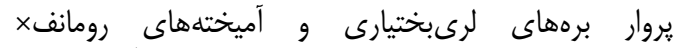

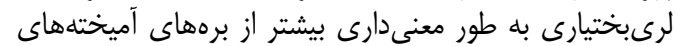

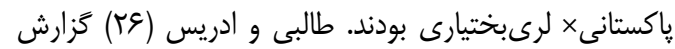

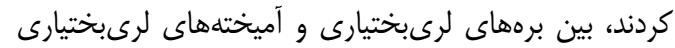

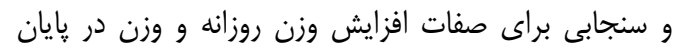

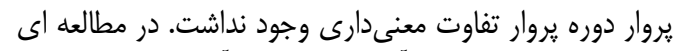

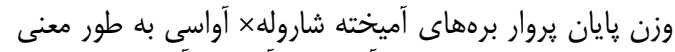

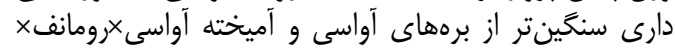

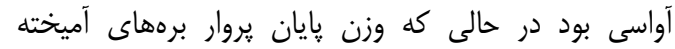

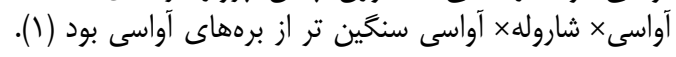

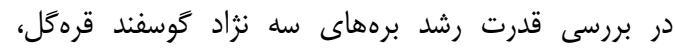


ناشى از سرعت رشد بيشتر برههاى نر در مقايسه با برهاى

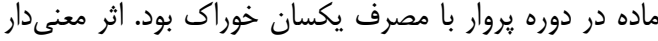

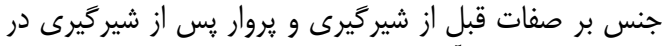

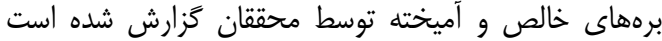

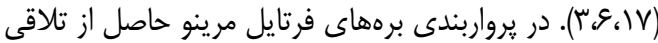

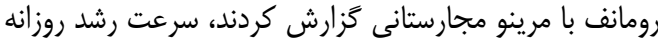

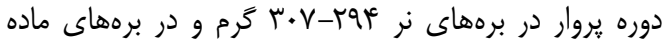

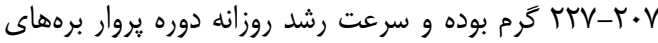

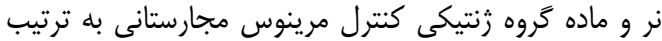

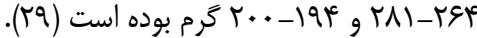
در تركيبهاى زنتيكى متفاوت اثر سال بران بر صغات

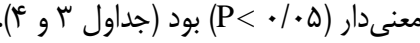

كزارش كردند كه اثر سال و گروها زنتيكى بر متوسط افزايش

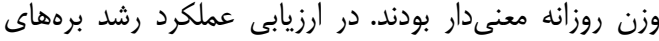

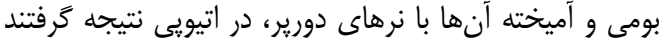

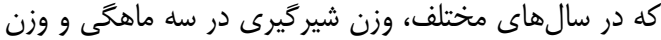

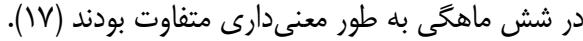
جنس تاثير معنى دارى بر صفات قباري

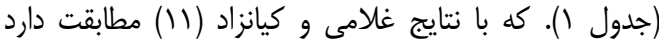

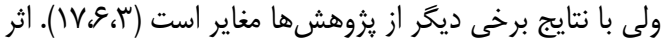

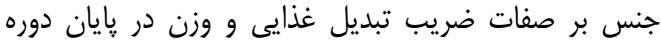

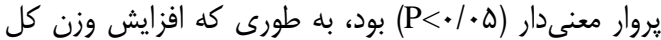

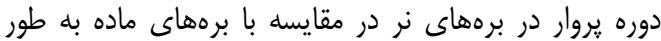

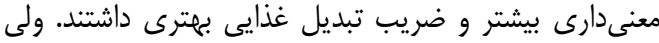

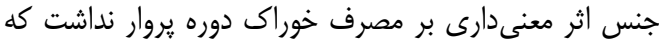

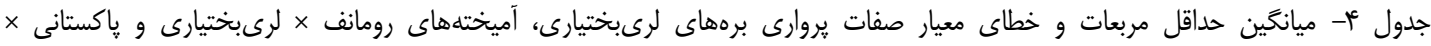
لرى ميختيارى تحت تاثير اثرات متقابل

Table 4. Least square means and standard error of feedlot traits of Lori-Bakhtiari, Romanov $\times$ Lori-Bakhtiari and Pakistani $\times$ Lori-Bakhtiari crossbred lambs influenced by interaction effects

\begin{tabular}{|c|c|c|c|c|c|c|c|c|}
\hline بازده غذايى & ضريب تبديل دوره & مصرف خوراك & 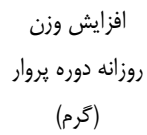 & افزوايش وزن دوره (كيلوكَم) & تعداد & سال & جنس & نزراد \\
\hline $1 . / 9 \Lambda \pm \cdot / / V^{\mathrm{de}}$ & $q / \widetilde{T} \pm \cdot / V \cdot{ }^{b c}$ & $1 / r r \pm \cdot / \cdot r^{\mathrm{e}}$ & $|r| \pm \mid r^{\mathrm{e}}$ & $11 / 99 \pm 1 / \cdot 0^{\mathrm{e}}$ & 1. & اول & \multirow{2}{*}{ ماده } & \multirow{4}{*}{ لرى بختيارى } \\
\hline $11 / \varepsilon \cdot \pm 1 / r \omega^{\text {cde }}$ & $N / T F \pm 1 / / \Gamma^{\mathrm{cd}}$ & $r / \cdot r \pm \cdot / \cdot r^{a b}$ & $r r \Delta \pm 19^{a b}$ & $r / / \Lambda \pm \pm / / r^{a b}$ & r & دوم & & \\
\hline $\mid Q / \Delta r \pm \cdot / V r^{a}$ & $\varepsilon / 9 V \pm \cdot \mid q q^{\mathrm{d}}$ & $1 / \pi \pm \cdot 1 \cdot r^{\mathrm{e}}$ & $1 / 9 \pm 11^{c d}$ & $\mid \varepsilon / A r \pm 1 / . .^{c d}$ & 1. & اول اول & \multirow[b]{2}{*}{ نر } & \\
\hline $\mid r / \cdot r \pm \cdot / g \cdot b c$ & $\checkmark / \wedge \Delta \pm \cdot / \Delta \digamma^{\mathrm{cd}}$ & $r / \cdot v \pm \cdot / \cdot r^{a}$ & $r q V \pm q^{a}$ & $r F / \cdot r \pm \cdot / \Lambda r^{a}$ & 10 & دوم & & \\
\hline$V / \Delta \cdot \pm 1 / \cdot r^{f}$ & $1 \% / 9 \cdot \pm \cdot /\left.9\right|^{\mathrm{a}}$ & $1 / \cdot 9 \pm \cdot / \cdot r^{f}$ & $1 \cdot \pm 18^{f}$ & $V / / \Delta \pm|/ F|^{f}$ & $\Delta$ & اول & ماده & \multirow{3}{*}{ باكتنانى × لرى } \\
\hline $10 / v \cdot \pm 1 / \sim^{a b}$ & $g / V r \pm 1 / r r^{c d}$ & $1 / 1 \Delta \pm \cdot / \cdot e^{\mathrm{ef}}$ & $|V \Delta \pm r|^{\text {cde }}$ & $1 \Delta / \Delta F \pm 1 / \Lambda \Delta^{\mathrm{cde}}$ & $r$ & اول & & \\
\hline $\mid r / / \Delta \pm 1 / / q^{\text {cde }}$ & $N / r \cdot \pm 1 / \cdot \Delta^{\mathrm{cd}}$ & $1 / v \wedge \pm \cdot / \cdot r^{\mathrm{d}}$ & $r \mid r \pm \Lambda^{b c}$ & $19 / 1 V \pm 1 / \Delta \Lambda^{b c}$ & r & دوم & نر & \\
\hline $11 / \Delta T \pm \cdot / q r^{\text {cde }}$ & $\Lambda / A F^{\mathrm{C}} \pm \cdot / \Lambda \varepsilon^{\mathrm{C}}$ & $1 / / V \pm \cdot / \cdot r^{\mathrm{e}}$ & $\left\|F^{f} \pm\right\| k^{e}$ & $11 / 9 r \pm 1 / r q^{e}$ & 9 & اول & \multirow{2}{*}{ ماده } & \multirow{4}{*}{ بختيارى × رومانف لرى } \\
\hline$q / \propto q \pm \cdot / v \cdot{ }^{\text {ef }}$ & 1./৭६ $\pm \cdot / q \mu^{\mathrm{b}}$ & $1 / q q^{c} \pm \cdot / \cdot r^{c}$ & $|\Lambda| \pm\left. 1\right|^{\mathrm{cd}}$ & $19 / \pi V \pm \cdot / 9 \Delta^{c d}$ & 11 & دوم & & \\
\hline $1 T / V q \pm \cdot / 9 q^{b c d}$ & $\wedge / R \& \pm \cdot / \wedge q^{c d}$ & $1 / r r \pm \cdot / \cdot r^{e}$ & $19 \cdot \pm 10^{\mathrm{de}}$ & $\mid f / r g \pm 1 / \mu F^{\mathrm{de}}$ & 9 & اول اول & \multirow[b]{2}{*}{ ن } & \\
\hline $\mid r / \cdot f^{c} \pm \cdot / q^{2} b c$ & $N / \varphi \pm \cdot / \Delta \mathrm{r}^{\mathrm{cd}}$ & $1 / 99 \pm \cdot / \cdot r^{b c}$ & $r \Delta \Delta \pm 1 \cdot{ }^{a}$ & $r r / Q q \pm \cdot / N \mathcal{Q}^{\mathrm{a}}$ & 10 & دوم & & \\
\hline
\end{tabular}

نظر آمارى معنىدار نبود. در يثوهشى بيان شده است كه

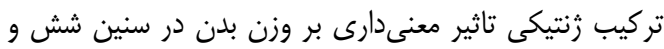

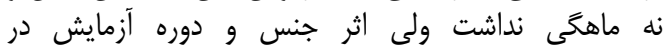

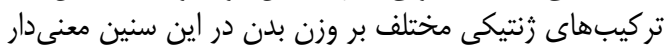

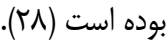

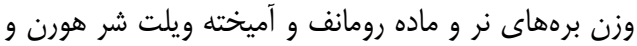

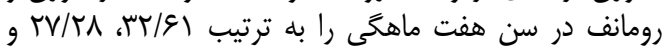

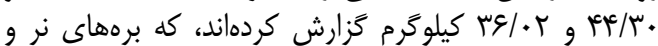

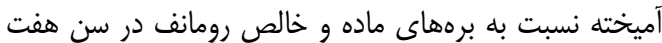

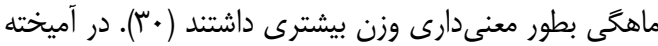

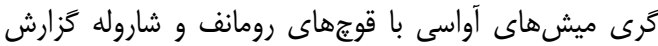

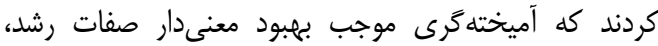
ضريب تبديل غذايى، درصد قطعات با ارزش لاشه و كاهش صنش
به طورى كه در سال اول در تركيبهاى زنتيكى مختلف رورائ

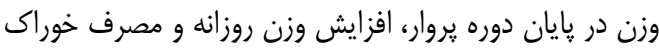

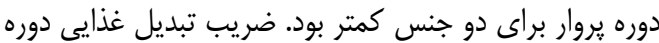

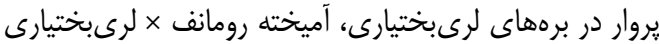

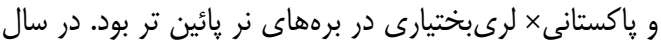

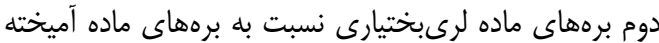

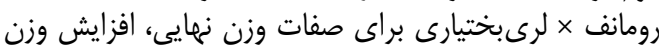

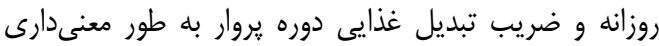

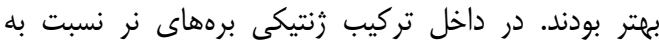

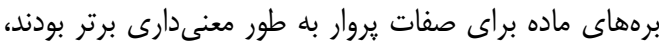

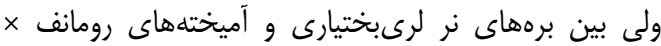

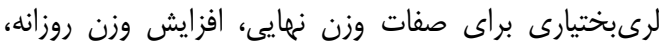
مصرف خوراك و ضريب تبديل غذايى دوره يروار، تفاوت از 


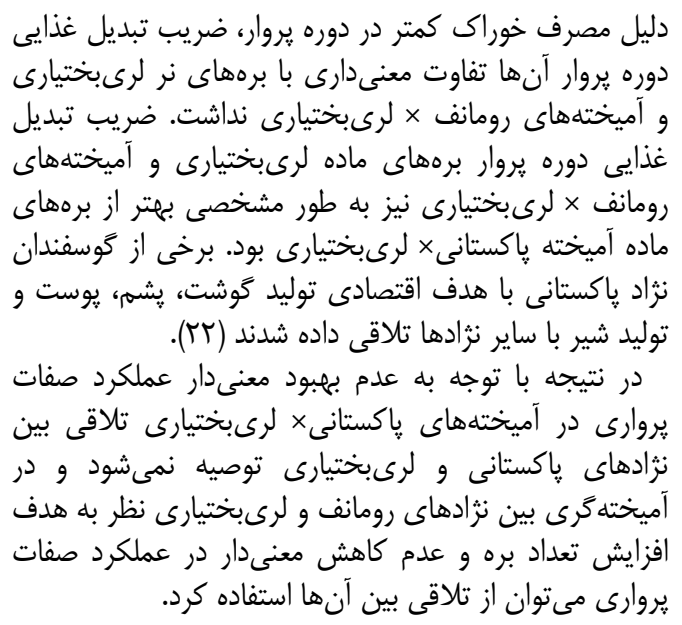

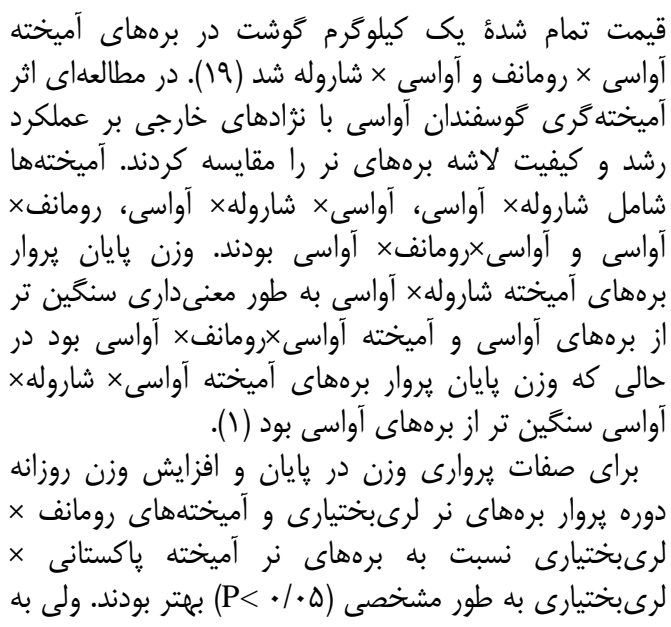

منابع

1. Abdullah, A.Y., I. Qudsieh Rasha and M. Nusairat Basheer. 2011. Effect of crossbreeding with exotic breeds on meat quality of Awassi lambs. Livestock Science, 142(1-3): 121-127.

2. Abdullah, A.Y., M. Momani Shaker, R.T. Kridli and I. Sada. 2003. Carcass composition of Awassi ram lambs in comparison to its crossbreds with Romanov and Charollais slaughtered at marketing age. Proceeding of $54^{\text {th }}$ EAAP European Association of Animal Production, Rome, Italy, 317pp.

3. Abdullah, A.Y., R.T. Kridli, M. Momani Shaker and M.D. Obeidat. 2010. Investigation of growth and carcass characteristics of pure and crossbred Awassi lambs. Small Ruminant Research, 94: 167175.

4. Boujenane, I. 2012. Productivity of Sardi, D'man and their crossbred ewes mated to terminal sires. Small Ruminant Research, 108: 22-27.

5. Castonguay, A., F. Minvielle and J.J. Dufour. 1990. Reproductive performance of Booroola x Finnsh Landrace and Booroola $x$ Suffolk ewe lambs, heterozygous for the $F$ gene and growth traits of their three-way cross lambs. Canadian Journal of Animal Science, 70: 55-65.

6. Cloete, J.J.E., S.W.P. Cloete, J.J. Olivier and L.C. Hoffman. 2007. Terminal crossbreeding of Dorper ewes to Ile de France, Merino Landsheep and SA Mutton Merino sires: Ewe production and lamb performance. Small Ruminant Research, 69: 28-35.

7. Costa, R.G., A.S.M. Batista, M.S. Madruga, S.G. Neto, R.C.R.E. Queiroga and J.T.A. Filho. 2009. Physical and chemical characterization of lamb meat from different genotypes submitted to diet with different fibre contents. Small Ruminant Research, 81: 29-34.

8. Dawson, L.E., R.A.F. Carson and L.O.W. McClinton. 2002. Comparison of productivity of Texel and Rouge de í Ouest ewes and their crosses. Animal Science, 75: 459-468.

9. Esmailizadeh, A.K., S.R. Miraei-Ashtiani, M.S. Mokhtari and M. Asadi Fozi. 2011. Growth performance of crossbred lambs and productivity of Kurdi Ewes as affected by the sire breed under extensive production system Journal of Agriculture Science and Technology, 13: 701-708.

10. Fahmy, M.H. 1989. Reproductive performance, growth and wool production of Romanov sheep in Canada. Small Ruminant Research, 2: 253-264.

11. Gholami, H. and M.R. Kianzad. 2014. Investigation of growth, carcass characteristics and economic efficiency of Zel breed and their crossbred Journal of Animal Production (Journal of Agriculture), 16(2): 137-145 (In Persian).

12. Güney O. 1990. Commercial crossbreeding between Ile-de-France, Rambouillet, Chios and local fattail Awassi for market lamb production Small Ruminant Research, 3: 449-456.

13. Hassan, A.H., S.T.M. Fahmy, M.T. Mousa and A.I.A. Suliman. 1996. The effect of crossbreeding between Chios and Ossimi sheep on growth performance and carcass characteristics of male lambs. Egypt Journal of Animal Production, 33: 61-70.

14. Hill, F.I., T. Ryan and A.F. Death. 1993. Awassi lamb performance in New Zealand quarantine Proc. N. Z. Soc. Animal Production, 53: 343-346.

15. Izadi far, J. and M. Dadpasand. 2009. Feedlot performance and carcass compositions of Ghezel and Mehraban lambs and their reciprocal crosses Iranian Journal of Animal Science, 40(1): 59-66 (In Persian).

16. Kuchtík, J., D. Zapletal and K. Šustová. 2012. Chemical and physical characteristics of lamb meat related to crossbreeding of Romanov ewes with Suffolk and Charollais sires. Meat Science, 90: 426430. 
17. Lakew, M., M. Haile-Melekot and G. Mekuriaw. 2014. Evaluation of growth performance of local and Dorper $\times$ local crossbred sheep in eastern Amhara region, Ethiopia. Iranian Journal of Applied Animal Science, 4(4): 787-794.

18. Makarechian, M., A. Farid and N. Sefidbakht. 1977. Lamb growth performance of Iranian fat-tailed Karakul, Mehraban and Naeini breeds of sheep and their crosses with Corriedale and Targhee rams. Animal Production, 25: 331-341.

19. Momani Shaker, M., A.Y. Abdullah, R.T. Kridli, I. Sada, R. Sovjak and M.M. Muwalla. 2002. Effect of crossing indigenous Awassi sheep breed with mutton and prolific sire breeds on the growth performance of lambs in a subtropical region. Czech Journal of Animal Science, 47(6): 239-246.

20. Momani Shaker, M., R.T. Kridli, A.Y. Abdullah, M. Malinova, S. Sanogo, I. Sada and D. Lukesova. 2010. Effect of crossbreeding European sheep breed with Awassi sheep on growth efficiency of lambs in Jordan. Agricultura Tropica Et Subtropica, 43(2): 127-133.

21. Phillips, W.A., M.A. Brown, H.G. Dolezal and G.Q. Fitch. 2005. Feedlot performance and carcass characteristics of lambs sired by Texel, Romanov, St. Croixor Dorset rams from Polypay and St. Croix ewes. Sheep and Goat Research, 20: 11-16.

22. Rasali, D.P., J.N.B. Shrestha and G.H. Crow. 2006. Development of composite sheep breeds in the world: A review. Canadian Journal of Animal Science, 86: 1-24.

23. SAS. 2010. Release 9.3, SAS Institute Inc., Cary, North Carolina, USA.

24. Stanford, K., G.L. Wallins, S.D.M. Jones and M.A. Price. 1998. Breeding Finnish Landrace and Romanov ewes with terminal sires for out-of-season market lamb production Small Ruminant Research, 27: 103-110.

25. Suarez, V.H., M.R. Busetti, C.A. Garriz, M.M. Gallinger and F.J. Babinec. 2000. Pre-weaning growth, carcass traits and sensory evaluation of Corriedale, CorriedalexPampinta and Pampinta lambs. Small Ruminant Research, 36: 85-89.

26. Talebi, M.A. and M.A. Edriss. 1997. Comparison feedlot and carcass characteristics of LoriBakhtiari and Sanjabi $\times$ Lori-Bakhtiari crossbred male lambs. Journal of Pajouhesh and Sazandegi, 34: 135-139 (In Persian)

27. Talebi, M.A., S.R. Miraei Ashtiani, M. Moradi Shahrbabak and A. Nejati Javaremi. 2008. Relationship between growth and carcass traits in Lori-Bakhtiari. Iranian Journal of Animal Science, 39(1): 29-37 (In Persian).

28. Thiruvenkadan, A.K., K. Karunanithi, M. Murugan, K. Arunachalam and R. Narendra Babu. 2009. A comparative study on growth performance of crossbred and purebred Mecheri sheep raised under dry land farming conditions. South African Journal of Animal Science, 39 (Supple 1): 121-125.

29. Veress, L. 1982. Improvement crossbreeding of Hungarian Merinos for increased prolificacy and milk yield. $33^{\text {th }}$ Annual Meeting of the EAAP Annual Meeting, Leningrad.

30. Zapasnikien , B. and R. Nainien . 2012. The effects of crossbreeding Romanov ewes with Wiltshire Horn rams on ewe fertility and progeny performance. Veterinarija ir zootechnika (Vet Med Zoot), 57(79): 72-76. 


\title{
Growth and Feedlot Performance of Lori-Bakhtiari, Romanov $\times$ Lori-Bakhtiari and Pakistani $\times$ Lori-Bakhtiari Crossbred Lambs
}

\author{
Mohammad Ali Talebi ${ }^{1}$ and Korush Gholamhosani ${ }^{2}$
}

1- Animal Science Research Department, Chaharmahal and Bakhtiari Agricultural and Natural Resources Research Center, AREEO, Shahrekord, Iran, (Corresponding Author: maitalebi@yahoo.com)

2- Deputy Animal Science, Organization of Agriculture Jahad Chaharmahal and Bakhtiari, Shahrekord

Received: December 6, 2015 Accepted: April 26, 2017

\begin{abstract}
This study was conducted to compare the feedlot performance and growth traits of LoriBakhtiari $(n=39)$, Romanov $\times$ Lori-Bakhtiari $(n=38)$ and Pakistani $\times$ Lori-Bakhtiari crossbred $(n=12)$ lambs during two consecutive years. Lambs were weaned at the age of $90 \pm 5$ days. After weaning and preliminary fattening period, three groups of lambs were fattened for 90 days in the same conditions. The results showed that weaning weight in Romanov $\times$ Lori-Bakhtiari crossbred lambs were significantly higher than in Lori-Bakhtiari and Pakistani $\times$ Lori-Bakhtiari crossbred lambs. Lori-Bakhtiari and Romanov $\times$ Lori-Bakhtiari lambs were not significantly different $(\mathrm{P}<0.05)$ for daily gain, feed intake, feed conversion ratio and final weight of the finishing period. Lori-Bakhtiari and Romanov $\times$ Lori-Bakhtiari lambs for daily gain (200 and $184 \mathrm{gr}$ ), feed conversion ratio (8.52 and 8.95) and final weight (51.78 and $50.26 \mathrm{~kg}$ ) of the finishing period were significantly $(\mathrm{P}<0.05)$ higher than in Pakistani $\times$ Lori-Bakhtiari crossbred lambs for this traits $(47.71 \mathrm{~kg}, 155 \mathrm{gr}$ and 11.07 , respectively). Effect of year in different genetic group on fattening traits was significant $(\mathrm{P}<0.05)$. In each genetic group between male and female lambs for fattening traits was significant differences $(\mathrm{P}<0.05)$, however, between Lori-Bakhtiari and Romanov $\times$ Lori-Bakhtiari lambs for fattening traits were not significantly different. Therefore, crossbreeding between Romanov and Lori-Bakhtiari were better than crossbreeding between Pakistani and Lori-Bakhtiari to improve growth and fattening performance.
\end{abstract}

Keywords: Crossbreeding, Growth, Feedlot, Lori-Bakhtiari, Romanov, Pakistani 